\title{
OVIPOSIC̣̃̃O, TAMANHO DE OVOS E MEDIDA DO COMPRIMENTO DA CONCHA EM DIFERENTES FASES DO DESENVOLVIMENTO DE SUBULINA OCTONA (BREGUIÉRE) (PULMONATA, SUBULINIDAE) EM CONDIC̣ÕES DE LABORATÓRIO
}

\author{
Elisabeth C. de Almeida Bessa 1,2 \\ José L. de Barros Araújo ${ }^{1}$
}

\begin{abstract}
SIZE OF EGGS AND MEASU IRIMMENT OF THE SHELL LENGHT IN DIFFERENT PHASES OF THE DESENVELOPMENT OF SUBULINA OCTONA (BRUGUIERE) (PULMONATA. SUBULINIDAF) UNDER LABORATORIAL. CONDTIONS. The study of 41 individually specimes of Subulina octona (Bruguiére, 1789) during 182 days indicated that oviposition occur between $10 \mathrm{pm}$ and $10 \mathrm{am}$. The interval between ovipositions was from seven to 31 days. In the eggs, the greatest axis was $1.5-2.0 \mathrm{~mm}$ while the smallest was $1.2-1.6 \mathrm{~mm}$. The lenght of shell of recently ecloded snails was $1.3-1.8 \mathrm{~mm}$. The growth rate of adult snails was measured and they grew more in the first 45 days than in the last 75 days subsequent of the study.

KEY WORDS. Pulmonata, Subulinidae, Subulina octona, oviposition, eggs, shell
\end{abstract}

Subulina octona (Bruguiére, 1789) é um molusco terrestre com ampla distribuição geográfica e freqüentemente encontrado na natureza (ARAÚJo 1982). A literatura concernente a esta espécie enfatiza principalmente aspectos conquiliológicos, sinonímia e distribuição geográfica (LA SAGRA 1853; TRYON \& PILSBRY 1906; Thiele 1931). No mais, os trabalhos referentes à $S$. octona, destacam aspectos da anatomia e histologia das partes moles (LANZIERI 1966; TILLIER 1989), morfologia do ovo e alguns aspectos comportamentais (VENMANS \& Fromming 1957; Marcus \& Marcus 1968; ARaúJo \& Bessa 1993).

Estudos que enfatizem aspectos morfológicos somados a dados biológicos, podem contribuir para um melhor conhecimento deste molusco já que, muitas vezes, formas jovens e mesmo os adultos de subulinídeos são confundidos entre sii pela semelhança da concha e pelo habitat preferencial (ARAÚJO 1982; DUTRA 1988).

Foram enfocados neste trabalho, aspectos sobre oviposição, tamanho dos ovos e comprimento da concha em diferentes fases do desenvolvimento da $S$. octona.

1) Departamento de Parasitologia. Instituto de Patologia Tropical e Saúde Pública, Universidade Federal de Goiás. 74605-050, Goiânia, Goiás, Brasil.

2) Bolsista do CNPq. 


\section{MATERIAL E MÉTODOS}

O presente trabalho foi desenvolvido no Laboratório de Malacologia da Universidade Federal Rural do Rio de Janeiro (Itaguaí, Rio de Janeiro).

A partir da criação matriz foram isolados 41 caracóis nos quais constatouse, através da transparência da concha, a presença dos primeiros ovos. Estes indivíduos foram mantidos isolados (um a um), por 182 dias, em caixas de plástico ( $9,0 \mathrm{~cm}$ de diâmetro e $5,8 \mathrm{~cm}$ de profundidade) contendo terra vegetal esterelizada $\left(120^{\circ} \mathrm{C}\right.$ por uma hora) até cerca da metade de sua altura. Diarimente esta terra foi umedecida com água, renovando-se também o alimento dos moluscos, que se constituía de alface (Lactuca sativa Linnaeus) mais ração concentrada (ração para pintos de corte e carbonato de cálcio, na proporção de 3:1). As caixas foram cobertas com tecido de algodão escaline preso com elástico de escritório.

Foram realizadas observações diárias, às 04:00, 10:00, 16:00 e 22:00 horas, até que todos os indivíduos realizassem a primeira oviposição. Com isso, foi possível determinar o horário preferido para a oviposição. Posteriormente as observações foram feitas a intervalos de 12 horas (06:00 e 18:00 horas), durante 182 dias (janeiro a julho de 1990), a fim de acompanhar o número e o intervalo entre as oviposições para os 41 exemplares.

Para determinar o tamanho médio dos ovos de $S$. octona foram medidos 180 ovos, ovipositados por 35 caracóis de mesma origem mantidos isolados um a um. Realizou-se, para cada ovo, a medida dos dois principais eixos, considerando que o ovo apresenta dois achatamentos paralelos.

Para conhecer o tamanho da concha a partir do momento de eclosão, utilizaram-se 32 moluscos recém-eclodidos, os quais foram mantidos isolados, um a um, durante 158 dias. Foram feitas observações diárias até a primeira constatação de ovo, para o primeiro indivíduo, quando as conchas dos 32 exemplares foram medidas. A partir de então, as medidas foram realizadas a intervalos de 15 dias até 158 dias após o isolamento dos jovens.

Todas as medidas foram executadas com o auxílio de um paquímetro Kanon (Mardened Stainless $1 / 28$ in $1 / 20 \mathrm{~mm}$ ). As observações de temperatura (máxima e mínima) e umidade relativa foram feitas diarimente.

\section{RESULTADOS E DISCUSSÃO}

Através do acompanhamento de 41 exemplares de $S$. octona mantidos isolados, observou-se que as oviposições dessa espécie ocorrem sempre no fundo da caixa, através de fendas na terra, abertas pelo caracol ao enterrar-se. Os ovos são depositados juntos e, na maioria da vezes, nas laterais da caixa, fato também observado para outros moluscos terrestres como Achatina fulica por PANSON \& CHASE (1984) e em Thaumastus taumaisi por JURBERG et al. (1988).

Conforme mostra a tabela $I$, as oviposições foram realizadas por todos os moluscos 15 dias após o isolamento dos indivíduos, no intervalo entre 22:00 e 10:00 horas, preferencialmente entre 22:00 e 04:00 horas. Tal atividade noturna foi referida para os Pulmonata terrestres por FreTter \& GraHAM (1964). Na 
tabela II pode ser observado o número de oviposições realizadas de janeiro a julho de 1990. Os maiores números de oviposições aconteceram em fevereiro, março e abril e o menor número no mês de janeiro. Este menor número pode ser explicado pelo fato das observações restringirem-se à última semana daquele mês. Os maiores números de oviposições para févereiro, março e abril em contraste com os menores observados nos meses de maio, junho e julho, podem ser explicados pela queda de temperatura ohservada nestes meses (Fig. 1), o que pode determinar uma menor atividade biológica para pulmonados terrestres (FRETTER \& GRAHAM 1964).

Tabela I. Horários de oviposições de Subulina octona, verificados a intervalos de seis horas em 41 exemplares mantidos isolados durante 15 dias em condiçōes de laboratório.

\begin{tabular}{ccc}
\hline $\begin{array}{c}\text { Periodo de observação } \\
\text { (horas) }\end{array}$ & Número de oviposições & Porcentagem \\
\hline $\begin{array}{r}4-10 \\
10-16\end{array}$ & 10 & 24,4 \\
$16-22$ & - & - \\
$22-4$ & - & 75,6 \\
\hline
\end{tabular}

Tabela II. Número total de oviposições, observados mensalmente em 41 exemplares de Subulina octona, mantidos isolados por 182 dias em condições de laboratório.

\begin{tabular}{cc}
\hline Período de observação & Número de posturas \\
\hline Janeiro & 25 \\
Fevereiro & 126 \\
Março & 114 \\
Abril & 123 \\
Maio & 93 \\
Junho & 62 \\
Julho & 64 \\
\hline
\end{tabular}

A figura 2 demonstra os intervalos (dias) entre uma oviposição e outra para S. octona. Observou-se que estes intervalos estão compreendidos entre sete e 31 dias. Cerca de $76 \%$ dos intervalos estão concentrados entre sete e 15 dias. Constatou-se que, após a postura, novos ovos foram observados no mesmo caracol no dia seguinte. Fato observado também, para a mesma espécie, por Marcus \& MARCUS 1968). no mais, na literatura mundial, não foram encontrados dados acerca do intervalo entre posturas para $S$. octona, podendo ser esta, uma característica do comportamento reprodutivo da espécie.

Os ovos de $S$. octona são esféricos e esbranquiçados, apresentando dois achatamentos paralelos (ARAúJo 1982). Estes ovos são envoltos por uma casca calcárea que segundo MARCUS \& MARCUS (1968), é proveniente de glândulas externas do espermoviduto. 

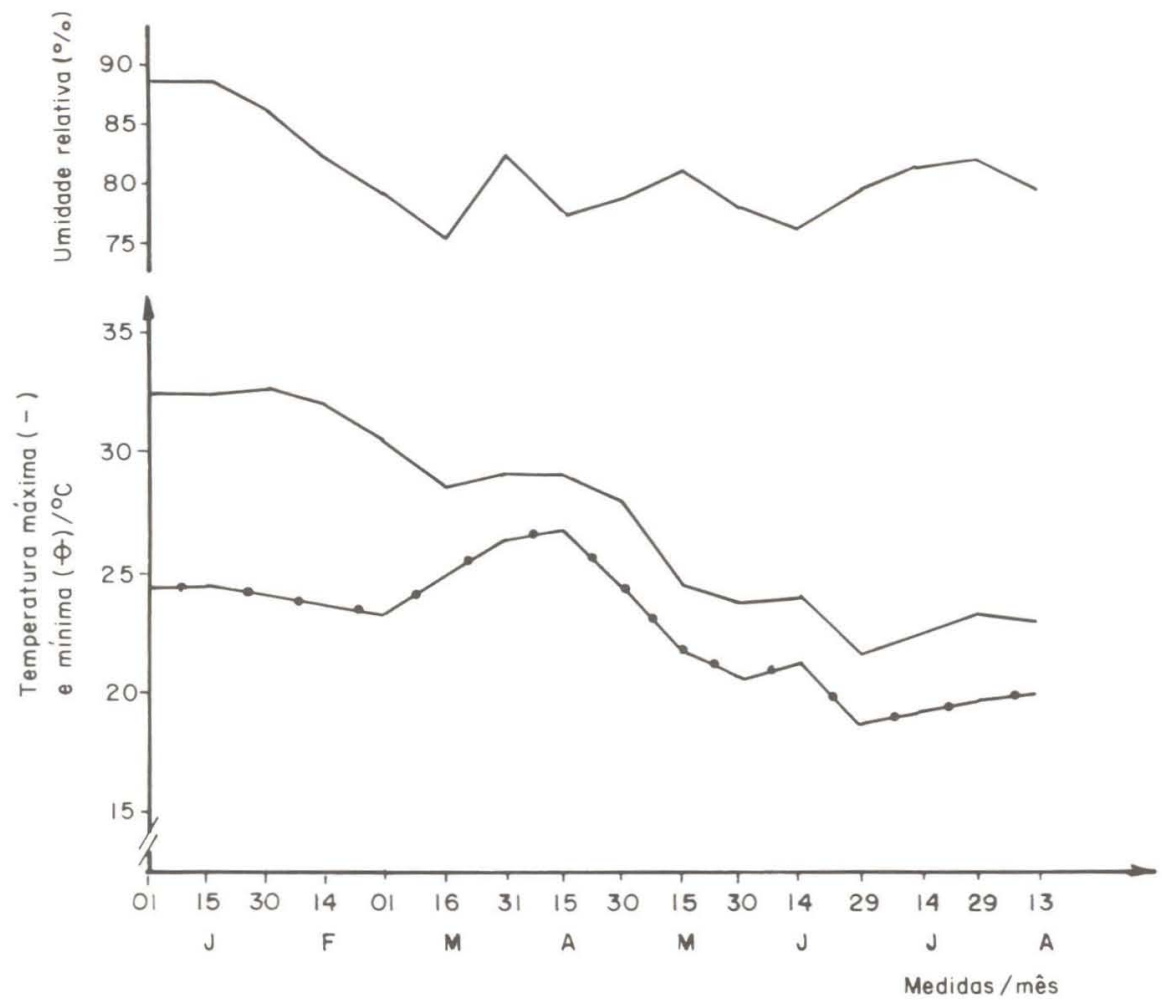

Fig. 1. Medidas quinzenais de temperatura (máxima e mínima) e umidade relativa do ar, observadas entre 01-I-1990 e 13-VIII-1990.

Como mostra a tabela III, pode ser observado que o ovo de $S$. octona mede de 1,5 a 2,0mm em seu maior eixo e de 1,2 a $1,6 \mathrm{~mm}$ em seu menor eixo. Estes valores estão compreendidos entre àqueles citados por TRYON \& PILSBRY (1906), MARCUS \& MARCUS (1968) e ARAÚJO (1982) que foram respectivamente 1,8x

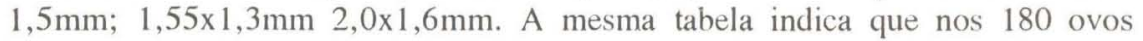
medidos, os valores mais freqüentes para as medidas do maior e menor eixo, foram 1,6 e $1,3 \mathrm{~mm}$, respectivamente.

A tabela IV demonstra o comprimento da concha de 32 jovens recémeclodidos e após o aparecimento do primeiro exemplar, entre estes, com ovos. Observou-se que a medida do comprimento da concha dos jovens após a eclosão variou entre 1,3 a $1,8 \mathrm{~mm}$. Tais valores são bastante semelhantes àqueles encontrados por MARCUS \& MARCUS (1968), que foram de $1,3 \mathrm{~mm}$ a $1,6 \mathrm{~mm}$. Verificouse que exemplares com comprimento de concha variando entre 9,1 e 13,5mm continham ovos. Observações semelhantes foram feitas por MARCUS \& MARCUS (1968) quando os mesmos, verificaram a presença de ovos em um exemplar de $S$. octona com 8,0mm de comprimento de concha. Segundo TRYON \& PILSBRY (1906) a reprodução em $S$. octona começa antes da concha atingir 2/3 do tamanho máximo da espécie. O comprimento da concha desta espécie, citado na literatura, varia de 


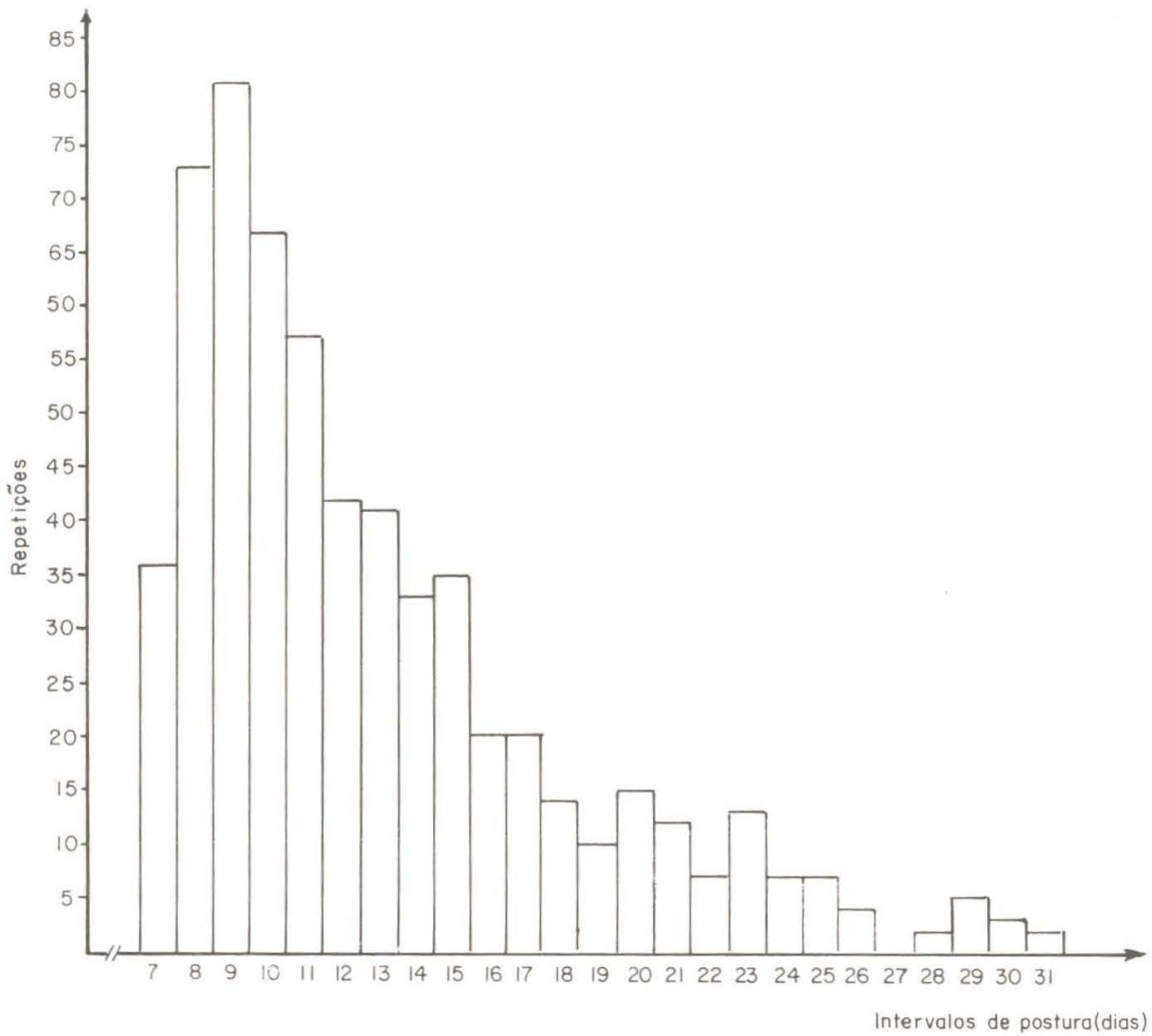

Fig. 2. Intervalos entre oviposições de 41 exemplares de Subulina octona mantidos isolados, observados entre 24-I-1990 e 24-VII-1990, sendo sete o número mínimo e 31 o máximo de dias entre oviposições.

Tabela III. Média, desvio padrão e coeficiente de variação dos eixos (maior e menor) de 180 ovos de Subulina octona, obtidos de exemplares mantidos isolados em condições de laboratório.

\begin{tabular}{|c|c|c|c|c|}
\hline & \multicolumn{2}{|c|}{ Eixo maior } & \multicolumn{2}{|c|}{ Eixo menor } \\
\hline & Comprimento (mm) & Número de ovos & Comprimento (mm) & Número de ovos \\
\hline & 2,00 & 10 & 1,60 & 15 \\
\hline & 1,80 & 21 & 1,50 & 9 \\
\hline & 1,70 & 31 & 1,40 & 52 \\
\hline & 1,60 & 65 & 1,30 & 84 \\
\hline & 1,50 & 53 & 1,20 & 20 \\
\hline $\bar{x}$ & 1,63 & & 1,35 & \\
\hline d & 0,13 & & 0,10 & \\
\hline CV $(\%)$ & 8,03 & & 7,62 & \\
\hline
\end{tabular}

*. (x) Média, (d) desvio padrão, (CV) coeficiente de variação. 
Tabela IV. Medidas do comprimento da concha de 32 exemplares de Subulina octona em seguida à eclosão e no aparecimento do primeiro exemplar com ovo, mantidos isolados em condições de laboratório.

\begin{tabular}{lrrrrr}
\hline \multirow{2}{*}{$\begin{array}{c}\text { Idade } \\
\text { fisiológica }\end{array}$} & \multicolumn{5}{c}{ Comprimento da concha (mm) } \\
\cline { 2 - 6 } & Maior & Menor & Moda & Média (t d) & CV (\%) \\
\hline Recém-eclodidos & 1,8 & 1,3 & 1,6 & $1,53(+0,169)$ & 11,04 \\
Primeiro exemplar com ovos & 13,5 & 9,1 & 11,0 & $11,36(+1,027)$ & 9,04 \\
\hline
\end{tabular}

*. (d) Desvio padrão, (CV) coeficiente de variação.

Tabela $V$. Medidas quinzenais dos comprimentos das conchas de 32 exemplares de Subulina octona mantidos isolados e observados por 120 dias, após o aparecimento do primeiro indivíduo com ovo, realizadas entre 07-IV-1990 e 04-VIII-1990 em condições de laboratório.

\begin{tabular}{ccccr}
\hline \multirow{2}{*}{$\begin{array}{c}\text { Período de } \\
\text { observação }\end{array}$} & Maior & Menor & Média $(+\mathrm{d})$ & CV $(\%)$ \\
\cline { 2 - 5 } & 13,5 & 9,1 & $10,25(+1,168)$ & 11,39 \\
\hline 07-IV & 15,0 & 9,9 & $12,55(+1,118)$ & 8,90 \\
21-IV & 16,3 & 11,0 & $14,11(+1,183)$ & 8,38 \\
O6-V & 17,1 & 12,5 & $14,98(+1,075)$ & 7,17 \\
21-V & 18,1 & 13,2 & $15,83(+1,058)$ & 6,68 \\
05-VI & 18,5 & 13,6 & $16,27(+1,035)$ & 6,36 \\
20-VI & 19,2 & 14,3 & $16,58(+0,990)$ & 5,97 \\
05-VII & 19,5 & 14,8 & $16,88(+0,996)$ & 5,90 \\
20-VII & 19,8 & 15,0 & $17,06(+1,026)$ & 6,01 \\
04-VIII & & & & \\
\hline
\end{tabular}

*. (d) Desvio padrão, (CV) coeficiente de variação.

24,0mm (D'ORBIGNy 1837), 25,0mm (LA SAgra 1853), de 12,0 a 19,0mm (TRYON \& PIlsbry 1906), de 8,0 a 24,0mm (ZILCH 1959) e 13,0mm (ARAú.JO 1982). Dois terços dos valores extremos $(8,0$ e $25,0 \mathrm{~mm})$ relatados acima, são valores semelhantes àqueles encontrados nas observações do presente trabalho.

O comprimento da concha dos 32 exemplares após o aparecimento do primeiro indivíduo com ovo, foi observado através de medidas realizadas quinzenalmente, de 07-IV a 04-VIII de 1990, e encontra-se demonstrado na tabela V. Oberservou-se ainda, que nos primeiros 45 dias do experimento, o crescimento da concha foi em torno de 0,8 a $1,5 \mathrm{~mm}$ e nos últimos dias os valores estiveram entre 0,2 e $1,0 \mathrm{~mm}$. Estes resultados concordam com a afirmação de VENMANS \& FROMMING (1957) de que, durante o desenvolvimento dos embriões, torna-se mais lento o crescimento da concha materna, o mesmo fato ocorrendo nos moluscos mais velhos. Tal observação também pode explicar o decréscimo nas taxas de crescimento após 45 dias do aparecimento de indivíduos com ovo, pois nesta data, virtualmente quase todos os indivíduos já apresentavam ovos. 
Marcus \& Marcus (1968) relataram o crecimento de um exemplar de $S$. octona de 16,9 para $20,0 \mathrm{~mm}$ em quatro meses de observação, mas a alimentação oferecida durante a observação não foi mencionada, o que dificulta uma comparação.

\section{REFERÊNCIAS BIBLIOGRÁFICAS}

ARAúJo, J.L. DE B. 1982. Alguns moluscos terrestres como hospedeiros intermediários de parasitos de animais domésticos, no Brasil: estudo sobre anatomia, sistemática e participação em Helmintoses. Tese de Doutorado, não publicada, Instituto de Biologia, Universidade Federal Rural do Rio de Janeiro, Itaguaí, 103p.

AraúJo, J.L. DE B. \& E.C. DE A. Bessa. 1993. Moluscos de importância econômica no Brasil. II Subulinidae. Subulina octona (Bruguiére) (Mollusea, Gastropoda, Pulmonata, Stylommatophora). Revta bras. Zool. 10 (3): 489-497.

D’Orbigny, A. 1837. Voyage dans l’Amerique Méridionale. Mollusques 5 (3): 185-376.

Dutra, A.V.C. 1988. Aspectos da ecologia e da reprodução de Leprinaria unilamellata (d'Orbiny, 1835) (Gastropoda, Subulinidae). Revta bras. Zool. 5 (4): $581-591$.

Fretter, V. \& A. Graham. 1964. Reproduction, p.127-156. In: K. M. Wilbur \& C.M. Yonge (eds). Physiology of Mollusca. New York, Academic Press, $473 p$.

Jurberg, P.; M.M. Barros; L.A. Gomes \& A.C. Dos S. Coelho. 1988. Superfamília Bulimuloidea do Brasil. Bulimulidae: Thaumastus (Thaumastus) taunaisi (Férussac, 1822), com dados biológicos e aspectos comportamentais (Mollusca, Gastropoda, Pulmonata). Bol. Mus. Nac. Zool. 317: 1-40.

La Sagra, M.R. 1853. Histórie Physique, politique et naturelle de l'Ile de Cuba. Paris, Arthur Bertrand, vol. I, 264p.

LANZIERI, P.D. 1966. Alguns aspectos morfo-estruturais do aparelho genital de Subulina octona (Bruguiére, 1792) (Gastropoda, Pulmonata, Subulinidae). Tese de Mestrado, não publicada, Instituto de Biologia, Universidade Federal Rural do Rio de Janeiro, Itaguaí, 44p.

Marcus, E. \& E. Marcus. 1968. Uber einige Subulidae (Pulmonata) von São Paulo. Beitr. Neotrop. Fauna 5: 186-208.

PANSON, P.A. \& R. CHASE. 1984. The life-cycle and reproductive activity of Achatina fulica (Bowdich) in laboratory culture. J. Moll. Stud. 50: 85-91.

THIELE, J. 1931. Handbuch der systematischen Weichtierkunde. Berlin, Jena, $778 p$.

TILliER, S. 1989. Comparative morfology, phylogeny and classification of land snails and slugs (Gastropoda: Pulmonata: Stylommatophora). Malacologia 30 $(1-3): 1-289$.

Tryon, G.W. \& M.A. Pilsbry. 1906. Manual of Conchology. Structural and 
systematic. Philadelphia, Academy of Natural Sciences, XIII, 357p.

VENMANS. L.A.W.C.\& E. FRomminG. 1957. Notes on the anatomy and biology of Subulina kassaiana Rochebrune \& Germain. Basteria 21 (1-2): 14-38.

Zilch, A. 1959/1960. Euthyneura, p. 129-710. In: W. WenZ (ed.). Gastropoda. Berlin, Gebrüder Borntraeger. vol. lï. 834p. 